\title{
On Behavior of the Periodic Orbits of a Hamiltonian System of Bifurcation of Limit Cycles
}

\author{
Amor Menaceur, ${ }^{1}$ Mohamed Abdalla $\mathbb{D D}^{2,3}$ Sahar Ahmed Idris $\mathbb{D D}^{4,5}$ and Ibrahim Mekawy ${ }^{6}$ \\ ${ }^{1}$ Laboratory of Analysis and Control of Differential Equations "ACED”, Department of Mathematics, University of Guelma, \\ P.O. Box 401, Guelma 24000, Algeria \\ ${ }^{2}$ Department of Mathematics, College of Science, King Khalid University, P.O. Box 9004, 61413 Abha, Saudi Arabia \\ ${ }^{3}$ Mathematics Department, Faculty of Science, South Valley University, Qena 83523, Egypt \\ ${ }^{4}$ College of Industrial Engineering, King Khalid University, Abha, Saudi Arabia \\ ${ }^{5}$ Department of Mathematics, Faculty of Science, Juba University-Khartoum, Juba, Sudan \\ ${ }^{6}$ Department of Mathematics, College of Sciences and Arts, ArRas, Qassim University, Saudi Arabia
}

Correspondence should be addressed to Sahar Ahmed Idris; sa6044690@gmail.com

Received 28 August 2021; Accepted 15 September 2021; Published 30 September 2021

Academic Editor: Sundarapandian Vaidyanathan

Copyright (c) 2021 Amor Menaceur et al. This is an open access article distributed under the Creative Commons Attribution License, which permits unrestricted use, distribution, and reproduction in any medium, provided the original work is properly cited.

In light of the previous recent studies by Jaume Llibre et al. that dealt with the finite cycles of generalized differential Kukles polynomial systems using the first- and second-order mean theorem such as (Nonlinear Anal., 74, 1261-1271, 2011) and (J. Dyn. Control Syst., vol. 21, 189-192, 2015), in this work, we provide upper bounds for the maximum number of limit cycles bifurcating from the periodic orbits of Hamiltonian system using the averaging theory of first order.

\section{Introduction}

Among the many interesting problems in the qualitative theory of planar polynomial differential systems is the study of their limit cycles (see $[1,2])$. In particular, concerning Kukles differential system of the form,

$$
\begin{aligned}
& \dot{x}=-y, \\
& \dot{y}=f(x, y),
\end{aligned}
$$

has a long history, where $f(x, y)$ is a polynomial with real coefficients of degree $n$. Since it was first introduced in Kukles 1944, many researchers have concentrated on its maximum number of limit cycles and their location. See, for example, [3-5].

In [6], Llibre and Mereu studied the maximum number of limit cycles using the averaging theory as follows:

$$
\left\{\begin{array}{l}
\dot{x}=y, \\
\dot{y}=-x-\sum_{k \geqslant 1} \varepsilon^{k}\left(f_{k}(x)+g_{k}(x) y+h_{k}(x) y^{2}+d_{k} y^{3}\right),
\end{array}\right.
$$

where, for every $k$, the polynomials $f_{k}(x), g_{k}(x)$, and $h_{k}(x)$ have degree $n_{1}, n_{2}$, and $n_{3}$, respectively. $d_{0}^{k} \neq 0$ is a real number and $\varepsilon$ is a small parameter.

Also, Makhlouf and Menaceur [7] studied the maximum number for the more generalized polynomial Kukles differential systems in the form

$$
\left\{\begin{array}{l}
\dot{x}=y, \\
\dot{y}=-x-\sum_{k \geqslant 1} \varepsilon^{k}\left(f_{k}(x)+g_{k}(x) y+h_{k}(x) y^{2}+g_{k}(x) y^{3}\right) .
\end{array}\right.
$$


The number of limit cycles bifurcating from the center $\dot{x}=-y^{2 p-1}$ and $\dot{y}=x^{2 q-1}$, where $p, q$ are positive integers, for the following two kinds of polynomial differential systems,

$$
\begin{aligned}
& \left\{\begin{array}{l}
\dot{x}=-y^{2 p-1}, \\
\dot{y}=x^{2 q-1}-\varepsilon f(x) y^{2 n-1},
\end{array}\right. \\
& \left\{\begin{array}{l}
\dot{x}=-y^{2 p-1}-\varepsilon p x f(x, y), \\
\dot{y}=x^{2 q-1}-\varepsilon q y f(x, y),
\end{array}\right.
\end{aligned}
$$

were investigated in the works $[8,9]$, respectively. In the current study, we discuss the maximum number of limit cycles of the following differential system:

$$
\left\{\begin{array}{l}
\dot{x}=-y^{2 p-1}, \\
\dot{y}=x^{2 q-1}-\varepsilon\left(f(x)+g(x) y^{2 n-1}+h(x) y^{2 n}+l(x) y^{2 n+1}\right),
\end{array}\right.
$$

where $p, q$, and $n$ are positive integers, the polynomials $f(x), g(x), h(x)$, and $l(x)$ have degree $n_{1}, n_{2}, n_{3}$, and $n_{4}$, respectively, and $\varepsilon$ is a small positive parameter. Clearly, system (5) with $\epsilon=0$ is an Hamiltonian system with

$$
H(x, y)=\frac{1}{2 q} x^{2 q}+\frac{1}{2 p} y^{2 p} .
$$

Our main theorems are given as follows.

Theorem 1. For the sufficiently small $|\varepsilon|$, system (5), using averaging theory of first order, has at most

$$
\max \left\{\left[\frac{n_{2}}{2}\right] p,\left[\frac{n_{4}}{2}\right] p+q\right\},
$$

The limit cycles bifurcating from the periodic orbits of the center are $\dot{x}=-y^{2 p-1}$ and $\dot{y}=x^{2 q-1}$, where [.] denotes the integer part function.

The proof of Theorem 1 is given in Section 3.

Theorem 2. Consider system (5) with $q=l p, l$ is a positive integer, and $|\varepsilon|$ sufficiently small; let $H\left(n_{i}, l\right)$ denote the maximum number of limit cycles of the polynomial differential system (5) bifurcating from the periodic orbits of the center $\dot{x}=-y^{2 p-1}$ and $\dot{y}=x^{2 l p-1}$ using the averaging theory of first order; then,

$$
\begin{aligned}
& \text { (a) } H\left(n_{i}, l\right)=\left[\frac{n_{2}}{2}\right]+\left[\frac{n_{4}}{2}\right]+1, \quad \text { if }\left[\frac{n_{2}}{2}\right]<l, \\
& \text { (b) } H\left(n_{i}, l\right)=\left[\frac{n_{4}}{2}\right]+l+1, \quad \text { if } l \leq\left[\frac{n_{2}}{2}\right] \leq l+\left[\frac{n_{4}}{2}\right], \\
& \text { (c) } H\left(n_{i}, l\right)=\left[\frac{n_{2}}{2}\right], \quad \text { if } l+\left[\frac{n_{4}}{2}\right]<\left[\frac{n_{2}}{2}\right] .
\end{aligned}
$$

The proof of Theorem 2 is given Section 4 .

\section{First-Order Averaging Method}

The averaging theory is an interesting method to research the limit cycles. Here, some specific function, associated to the initial system, is stated.

Theorem 3. The two initial value problems are as follows:

$$
\begin{aligned}
& \dot{x}=\varepsilon R(t, x)+\varepsilon^{2} G(t, x, \varepsilon), \quad x(0)=x_{0}, \\
& \dot{y}=\varepsilon f^{0}(y), \quad y(0)=x_{0},
\end{aligned}
$$

where $x, y$ and $x_{0} \in D$ which is an open domain of $\mathbb{R}$, $t \in[0, \infty), \varepsilon \in\left(0, \varepsilon_{0}\right], R$ and $G$ are periodic functions with their period $T$ with its variable $t$, and $f^{0}(y)$ is the average function of $R(t, y)$ with respect to $t$, i.e.,

$$
f^{0}(y)=\frac{1}{T} \int_{0}^{T} R(t, y) \mathrm{d} t .
$$

\section{Assume that}

(i) $R, \partial R / \partial x, \partial^{2} R / \partial x^{2}, G$, and $\partial G / \partial x$ are well defined, continuous, and bounded by a constant independent by $\varepsilon \in\left(0, \varepsilon_{0}\right]$ in $[0, \infty) \times D$.

(ii) $T$ is a constant independent of $\varepsilon$.

(iii) $y(t)$ belongs to $D$ on the time scale $1 / \varepsilon$. Then, the following statements hold:

(i) On the time scale $1 / \varepsilon$, we have

$$
x(t)-y(t)=O(\varepsilon) \text {, as } \varepsilon \longrightarrow 0 .
$$

(ii) If $p$ is an equilibrium point of the averaged system (10), such that

$$
\left.\frac{\partial f^{0}}{\partial y}\right|_{y=p} \neq 0
$$

$$
\text { then system (9) has a T-periodic solution }
$$
$\phi(t, \varepsilon) \longrightarrow p$ as $\varepsilon \longrightarrow 0$.

(iii) If (11) is a negative, therefore, the corresponding periodic solution $\phi(t, \varepsilon)$ of equation (9) according to $(t, x)$ is asymptotically stable, for all $\varepsilon$ sufficiently small; if (11) is a positive, then it is unstable.

For more information about the averaging theory, see [10-12].

\section{Proof of Theorem 1}

Here, we need to transform system (5) to the canonical from (9). Doing the change of $(p, q)$-polar coordinates $x=r^{p} \operatorname{Cs} \theta$ and $y=r^{q} \operatorname{Sn} \theta$ (see Appendix) and taking $\theta$ as an independent variable, then system (5) can be written as 


$$
\left\{\begin{array}{c}
\dot{r}=-\varepsilon r^{-q+1}(\operatorname{Sn} \theta)^{2 p-1}\left[\begin{array}{c}
f\left(r^{p} \operatorname{Cs} \theta\right)+g\left(r^{p} \operatorname{Cs} \theta\right)(\operatorname{Sn} \theta)^{2 n-1} r^{q(2 n-1)} \\
+h\left(r^{p} \operatorname{Cs} \theta\right)(\operatorname{Sn} \theta)^{2 n} \\
r^{2 q n}+l\left(r^{p} \operatorname{Cs} \theta\right)(\operatorname{Sn} \theta)^{2 n+1} r^{q(2 n+1)}
\end{array}\right], \\
\dot{\theta}=r^{p q-p-q}-\varepsilon p r^{-q} \operatorname{Cs} \theta\left[\begin{array}{c}
f\left(r^{p} \operatorname{Cs} \theta\right)+g\left(r^{p} \operatorname{Cs} \theta\right)(\operatorname{Sn} \theta)^{2 n-1} r^{q(2 n-1)} \\
+h\left(r^{p} \operatorname{Cs} \theta\right)(\operatorname{Sn} \theta)^{2 n} r^{2 q n} \\
+l\left(r^{p} \operatorname{Cs} \theta\right)(\operatorname{Sn} \theta)^{2 n+1} r^{q(2 n+1)}
\end{array}\right]
\end{array}\right.
$$

If we write then system (14) becomes

$$
\begin{aligned}
& f(x)=\sum_{k=0}^{n_{1}} a_{k} x^{k}, \\
& g(x)=\sum_{k=0}^{n_{2}} b_{k} x^{k}, \\
& h(x)=\sum_{k=0}^{n_{3}} c_{k} x^{k}, \\
& l(x)=\sum_{k=0}^{n_{4}} d_{k} x^{k},
\end{aligned}
$$

$$
\left\{\begin{array}{l}
\dot{r}=-\varepsilon r^{-q+1}\left[\sum_{k=0}^{n_{1}} a_{k}(C s \theta)^{k}(\operatorname{Sn} \theta)^{2 p-1} r^{p k}+\sum_{k=0}^{n_{2}} b_{k}(C s \theta)^{k}(\operatorname{Sn} \theta)^{2(p+n-1)} r^{p k+q(2 n-1)}\right. \\
\left.+\sum_{k=0}^{n_{3}} c_{k}(\operatorname{Cs} \theta)^{k}(\operatorname{Sn} \theta)^{2(p+n)-1} r^{p k+2 q n}+\sum_{k=0}^{n_{4}} d_{k}(\operatorname{Cs} \theta)^{k}(\operatorname{Sn} \theta)^{2(p+n)} r^{p k+q(2 n+1)}\right], \\
\dot{\theta}=r^{p q-p-q}-\varepsilon p r^{-q}\left[\sum_{k=0}^{n_{1}} a_{k}(C s \theta)^{k+1} r^{p k}+\sum_{k=0}^{n_{2}} b_{k}(C s \theta)^{k+1}(\operatorname{Sn} \theta)^{2 n-1} r^{p k+q(2 n-1)}\right. \\
\left.+\sum_{k=0}^{n_{3}} c_{k}(\operatorname{Cs} \theta)^{k+1}(\operatorname{Sn} \theta)^{2 n} r^{p k+2 q n}+\sum_{k=0}^{n_{4}} d_{k}(\operatorname{Cs} \theta)^{k+1}(\operatorname{Sn} \theta)^{2 n+1} r^{p k+q(2 n+1)}\right] .
\end{array}\right.
$$

where $\theta$ is the independent variable we get from system (16). From

$$
\frac{\mathrm{d} r}{\mathrm{~d} \theta}=\varepsilon R(r, \theta)+O\left(\varepsilon^{2}\right)
$$

where

$$
R(r, \theta)=-r^{-p q+p+1}\left[\begin{array}{c}
\sum_{k=0}^{n_{1}} a_{k}(C s \theta)^{k}(\operatorname{Sn} \theta)^{2 p-1} r^{p k}+\sum_{k=0}^{n_{2}} b_{k}(\operatorname{Cs} \theta)^{k}(\operatorname{Sn} \theta)^{2(p+n-1)} r^{p k+q(2 n-1)} \\
+\sum_{k=0}^{n_{3}} c_{k}(\operatorname{Cs} \theta)^{k}(\operatorname{Sn} \theta)^{2(p+n)-1} r^{p k+2 q n}+\sum_{k=0}^{n_{4}} d_{k}(\operatorname{Cs} \theta)^{k}(\operatorname{Sn} \theta)^{2(p+n)} r^{p k+q(2 n+1)}
\end{array}\right] .
$$
have

According to the notation introduced in Section 2, we 


$$
f^{0}(r)=-\frac{r^{-p q+p+1}}{T}\left[\begin{array}{c}
\sum_{k=0}^{n_{1}} a_{k} r^{p k} \int_{0}^{T}(C s \theta)^{k}(\operatorname{Sn} \theta)^{2 p-1} \mathrm{~d} \theta \\
+\sum_{k=0}^{n_{2}} b_{k} r^{p k+q(2 n-1)} \int_{0}^{T}(\operatorname{Cs} \theta)^{k}(\operatorname{Sn} \theta)^{2(p+n-1)} \mathrm{d} \theta \\
+\sum_{k=0}^{n_{3}} c_{k} r^{p k+2 q n} \int_{0}^{T}(C s \theta)^{k}(\operatorname{Sn} \theta)^{2(p+n)-1} \mathrm{~d} \theta \\
+\sum_{k=0}^{n_{4}} d_{k} r^{p k+q(2 n+1)} \int_{0}^{T}(\operatorname{Cs} \theta)^{k}(\operatorname{Sn} \theta)^{2(p+n)} \mathrm{d} \theta
\end{array}\right],
$$

and we write

$$
f^{0}(r)=-\frac{r^{-p q+p+1}}{T}\left[\begin{array}{c}
\sum_{k=0}^{n_{1}} a_{k} I_{k, 2 p-1} r^{p k}+\sum_{k=0}^{n_{2}} b_{k} I_{k, 2(p+n-1)} r^{p k+q(2 n-1)} \\
+\sum_{k=0}^{n_{3}} c_{k} I_{k, 2(p+n)-1} r^{p k+2 q n}+\sum_{k=0}^{n_{4}} d_{k} I_{k, 2(p+n)} r^{p k+q(2 n+1)}
\end{array}\right]
$$

where

$$
\begin{aligned}
& I_{i, j}=0, \text { if } i \text { or } j \text { is odd, } \\
& I_{i, j}>0, \text { if } i \text { and } j \text { are even. }
\end{aligned}
$$

$$
I_{i, j}=\int_{0}^{T} C s^{i} \theta S n^{j} \theta \mathrm{d} \theta
$$

Hence,

It is known that

$$
f^{0}(r)=-\frac{r^{q(2 n-p-1)+p+1}}{T}\left[\sum_{\substack{k=0 \\ k \text { even }}} n_{2} b_{k} I_{k, 2(p+n-1)} r^{p k}+\sum_{\substack{k=0 \\ k \text { keven }}} n_{4} d_{k} I_{k, 2(p+n)} r^{p k+2 q}\right],
$$

we obtain

$$
\begin{aligned}
& f^{0}(r)=-\frac{r^{q(2 n-p-1)+p+1}}{T}\left[\sum_{s=0}^{\left[n_{2} / 2\right]} b_{2 s} I_{2 s, 2(p+n-1)} r^{2 p s}\right. \\
& \left.+\sum_{s=0}^{\left[n_{4} / 2\right]} d_{2 s} I_{2 s, 2(p+n)} r^{2(p s+q)}\right] \text {. }
\end{aligned}
$$

For the simplicity of calculation, let $B_{s}=b_{2 s} I_{2 s, 2(p+n-1)}$ and $D_{s}=d_{2 s} I_{2 s, 2(p+n)}$; therefore, (24) can be reduced to

$$
f^{0}(r)=-\frac{r^{q(2 n-p-1)+p+1}}{T}\left[\sum_{s=0}^{\left[n_{2} / 2\right]} B_{s} r^{2 p s}+\sum_{s=0}^{\left[n_{4} / 2\right]} D_{s} r^{2(p s+q)}\right] \text {. }
$$

As we all know, the number of positive roots of $f^{0}(r)$ is equal to that of

$$
N(r)=\sum_{s=0}^{\left[n_{2} / 2\right]} B_{s} r^{2 p s}+\sum_{s=0}^{\left[n_{4} / 2\right]} D_{s} r^{2(p s+q)}
$$

Then, to find the real positive roots of $N(r)$, we must find the zeros of a polynomial in the variable $\rho=r^{2}$ :

$$
M(\rho)=\sum_{s=0}^{\left[n_{2} / 2\right]} B_{s} \rho^{p s}+\sum_{s=0}^{\left[n_{4} / 2\right]} D_{s} \rho^{p s+q} .
$$

So, the degree of $M(\rho)$ is bounded by $\mu=\max \left\{\left[n_{2} / 2\right] p,\left[n_{4} / 2\right] p+q\right\}$, we conclude that $f^{0}(r)$ has at most $\mu$ positive root $r$. Hence, Theorem 1 is proved.

\section{Proof of Theorem 2}

Consider the polynomial differential system (5) with $q=l p$; from equation (25) we obtain 


$$
f^{0}(r)=-\frac{r^{l p(2 n-p-1)+p+1}}{T}\left[\sum_{s=0}^{\left[n_{2} / 2\right]} B_{s} r^{2 p s}+\sum_{s=0}^{\left[n_{4} / 2\right]} D_{s} r^{2 p(s+l)}\right] .
$$

As we all know, the number of positive roots of $f^{0}(r)$ is equal to that of

$$
\begin{aligned}
& G(r)=B_{0}+B_{1} r^{2 p}+B_{2} r^{4 p}+\cdots+B_{\left[n_{2} / 2\right]} r^{2 p\left[n_{2} / 2\right]} \\
& +D_{0} r^{2 p l}+D_{1} r^{2 p(l+1)}+D_{2} r^{2 p(l+2)}+\cdots \\
& +D_{\left[n_{4} / 2\right]} r^{2 p\left(l+\left[n_{4} / 2\right]\right)} .
\end{aligned}
$$

To find the number of positive roots of polynomials $G(r)$, we distinguish 3 cases.

$$
f^{0}(r)=-\frac{r^{6 n-4}}{T}\left(B_{0}+B_{1} r^{2}+B_{2} r^{4}+D_{0} r^{6}+D_{1} r^{8}+D_{2} r^{10}\right),
$$

where $B_{s}=b_{2 s} I_{2 s, 2}$ and $D_{s}=d_{2 s} I_{2 s, 4}$. Using (A.3) of the Appendix, we obtain

$$
\begin{aligned}
& I_{0,2}=2.1033, \\
& I_{2,2}=0.60460, \\
& I_{4,2}=0.32339, \\
& I_{0,4}=0.63098, \\
& I_{2,4}=0.15115, \\
& I_{4,4}=6.9298 \times 10^{-2} .
\end{aligned}
$$

Case 1. For $\left[n_{2} / 2\right]<l$, the number terms in polynomial (29) is $\left[n_{2} / 2\right]+\left[n_{4} / 2\right]+2$. Now, we shall apply the Descartes theorem of the Appendix, we can choose the appropriate coefficients $B_{i}$ and $D_{j}$ so that the simple positive roots' number of $G(r)$ is at most $\left[n_{2} / 2\right]+\left[n_{4} / 2\right]+1$. Hence, (a) of Theorem 2 is proved.

Case 2. For $l \leq\left[n_{2} / 2\right] \leq l+\left[n_{4} / 2\right]$, the number terms in polynomial (29) is

$$
\left[\frac{n_{2}}{2}\right]+\left[\frac{n_{4}}{2}\right]+2-\left(\left[\frac{n_{2}}{2}\right]-l+1\right)=\left[\frac{n_{4}}{2}\right]+l+1 .
$$

By Descartes Theorem, we can choose the appropriate coefficients $B_{i}$ and $D_{j}$ so that the simple positive roots' number of $G(r)$ is at most $\left[n_{4} / 2\right]+l$. Hence, (b) of Theorem 2 is proved.

Case 3. For $l+\left[n_{4} / 2\right]<\left[n_{2} / 2\right]$, the number terms in polynomial (29) is $\left[n_{2} / 2\right]+1$; by Descartes Theorem, we can choose the appropriate coefficients $B_{i}$ and $D_{j}$ so that the simple positive roots number of $G(r)$ is at most $\left[n_{2} / 2\right]$. Hence, (c) of Theorem 2 is proved.

Example 1. We consider system (5), with $p=1, q=3, n=1$, and

$$
\begin{aligned}
& f(x)=\sum_{k=0}^{2} a_{k} x^{k}, \\
& g(x)=\sum_{k=0}^{4} b_{k} x^{k}, \\
& h(x)=\sum_{k=0}^{2} c_{k} x^{k}, \\
& l(x)=\sum_{k=0}^{4} d_{k} x^{k},
\end{aligned}
$$

where

$$
\begin{aligned}
& a_{0}=1, \\
& a_{1}=2.3, \\
& a_{2}=4.7, \\
& b_{0}=-0.5, \\
& b_{1}=1.1, \\
& b_{2}=6.3, \\
& b_{3}=2.5, \\
& b_{4}=-15.32, \\
& c_{0}=2.2, \\
& c_{1}=-6.4, \\
& c_{2}=7.3, \\
& d_{0}=4.65, \\
& d_{1}=3.4, \\
& d_{2}=-5.24, \\
& d_{3}=6.4, \\
& d_{4}=1.13 .
\end{aligned}
$$

In this case, $\operatorname{Cs} \theta$ and $\operatorname{Sn} \theta$ are T-periodic function with period $T=8.4131$. From equation (28), we obtain

So,

$$
f^{0}(r)=-\frac{r^{2}}{T}\left[\begin{array}{c}
-1.0517+3.8083 r^{2}-4.9553 r^{4} \\
+2.9320 r^{6}-0.79257 r^{8}+7.8473 \times 10^{-2} r^{10}
\end{array}\right] \text {. }
$$

This polynomial has four positive real roots: $r_{1}=0.6, r_{2}=0.8, r_{3}=1.1, r_{4}=1.3$, and $r_{5}=2$. According to statement (a) of Theorem 2, the system has exactly 5 limit cycles bifurcating from the periodic orbits of the center $\dot{x}=$ $-y$ and $\dot{y}=x^{5}$, using the averaging theory of first order.

\section{Conclusion}

In this work, by using averaging theory of the first order, we have proved upper bounds for the maximum number of limit cycles bifurcating from the periodic orbits of the Hamiltonian system. In addition, in the next work, a new 
condition with a new method will be used to prove our main result in this study.

\section{Appendix}

\section{1- $p, q)$-Polar Coordinates}

Following Lyapunov [13], we introduce the $(p, q)$-trigonometric functions $u(\theta)=\operatorname{Cs} \theta$ and $v(\theta)=\operatorname{Sn} \theta$ as the solution of the following initial value problem:

$$
\begin{aligned}
\dot{u} & =-v^{2 p-1}, \\
\dot{v} & =u^{2 q-1}, \\
u(0) & =\sqrt[2 q]{\frac{1}{p}}, \\
v(0) & =0 .
\end{aligned}
$$

Moreover, they satisfy the following properties:

(i) The functions $\operatorname{Cs} \theta$ and $\operatorname{Sn} \theta$ are $T$-periodic with

$$
T=2 p^{-1 / 2 q} q^{-1 / 2 p} \frac{\Gamma(1 / 2 p) \Gamma(1 / 2 q)}{\Gamma((1 / 2 p)+(1 / 2 q))},
$$

where $\Gamma$ is the gamma function.

(ii) For $p=q=1$, we have $\operatorname{Cs} \theta=\cos \theta$ and $\operatorname{Sn} \theta=\sin \theta$.

(iii) $p C s^{2} p \theta+q S n^{2 q} \theta=1$.

(iv) Let $\operatorname{Cs} \theta$ and $\operatorname{Sn} \theta$ be the (1.q)-trigonometrical functions, for $i$ and $j$ are both even (see [1]):

$$
I_{i ; j}=\int_{0}^{T} C s^{i} \theta S n^{j} \theta \mathrm{d} \theta=2 q^{-(j+1 / 2)} \frac{\Gamma(i+1 / 2 q) \Gamma(j+1 / 2)}{\Gamma((i+1 / 2 q)+(j+1 / 2))} .
$$

\section{2-Descartes Theorem}

The purpose of the Descartes theorem is to provide an insight on how many real roots a polynomial $P(x)$ may have.

Theorem A.1 (see [14]). Consider the real polynomial

$$
p(x)=a_{l_{1}} x_{l_{1}}+a_{l_{2}} x_{l_{2}}+\cdots+a_{l_{k}} x_{l_{k}},
$$

with $0 \leq l_{1}<l_{2}<\ldots<l_{k}$ and $a_{l_{i}} \neq 0$ real constants, for $i \in\{1,2,3, \ldots, k\}$. When $a_{l_{i}} a_{l_{i+1}}<0$, we say that $a_{l_{i}}$ and $a_{l_{i+1}}$ have a variation of sign. If the number of variations of signs is $n$, then $p(x)$ has at most $m$ positive real roots. Moreover, it is always possible to choose the coefficients of $p(x)$ in such a way that $p(x)$ has exactly $k-1$ positive real roots.

\section{Data Availability}

No data were used to support the study.

\section{Conflicts of Interest}

The authors declare that they have no conflicts of interest.

\section{Acknowledgments}

The third author extends appreciation to the Deanship of Scientific Research at King Khalid University for funding this work through Research Group Project, under Grant no. R.G.P-2/53/42.

\section{References}

[1] A. Gasull and J. Torregrosa, "A new algorithm for the computation of the Lyapunov constants for some degenerated critical points," Nonlinear Analysis: Theory, Methods \& Applications, vol. 47, no. 7, pp. 4479-4490, 2001.

[2] D. Hilbert, "Mathematische problems. lecture in: second international congress of mathematicians-Paris, France Nachrichten von der gesellschaft der wissenschaften $\mathrm{zu}$ göttingen, mathematisch-physikalische klasse," English Translation, vol. 5, pp. 253-297, 1900.

[3] J. Chavarriga, I. A. García, E. Szántó, and I. Sz ant o., "Limit cycles in Kukles systems of arbitrary degree with invariant ellipse," Nonlinear Analysis: Theory, Methods \& Applications, vol. 67, no. 4, pp. 1005-1014, 2007.

[4] J. M. Hill, N. G. Lloyd, and J. M. Pearson, "Centres and limit cycles for an extended Kukles system," The Electronic Journal of Differential Equations, vol. 119, pp. 1-23, 2007.

[5] A. Menaceur, S. Boulaaras, S. Alkhalaf, and S. Jain, "Limit cycles of a class of polynomial differential systems bifurcating from the periodic orbits of a linear center," Symmetry, vol. 12, no. 8, p. $15,2020$.

[6] J. Llibre and A. C. Mereu, "Limit cycles for generalized Kukles polynomial differential systems," Nonlinear Analysis: Theory, Methods \& Applications, vol. 74, no. 4, pp. 1261-1271, 2011.

[7] A. Makhlouf and A. Menaceur, "On the limit cycles of a class of generalized Kukles polynomial differential systems via averaging theory," International Journal of Differential Equations, vol. 2015, Article ID 325102, 10 pages, 2015.

[8] J. Llibre and A. Makhlouf, "Limit cycles of a class of generalized liénard polynomial equations," Journal of Dynamical and Control Systems, vol. 21, no. 2, pp. 189-192, 2015.

[9] A. Menaceur, S. Boulaaras, A. Makhlouf, K. Rajagobal, and M. Abdalla, "Limit cycles of a class of perturbed differential systems via the first-order averaging method," Complexity, vol. 2021, Article ID 5581423, 6 pages, 2021.

[10] A. Buică and J. Llibre, "Averaging methods for finding periodic orbits vai Brouwer degree," Bulletin des Sciences Mathematiques, vol. 128, pp. 7-22, 2004.

[11] J. A. Sanders and F. Verhulst, "Averaging methods in nonlinear dynamical systems," Applied Mathematical Science, Vol. 59, Springer-Verlag, New York, NY, USA, 1985.

[12] F. Verhulst, Nonlinear Differential Equations and Dynamical Systems. Universitex, Springer-Verlag, Berlin, 1996.

[13] A. M. Liapunov, "Stability of motion. with a contribution by V. A. Pliss and an introduction by V. P. Basov," Mathematics in Science and Engineering, Vol. 30, Academic Press, New York, London, 1966.

[14] I. S. Berezin and N. P. Zhidkov, Computing Methods, Pergamon, Oxford, UK, 1964. 